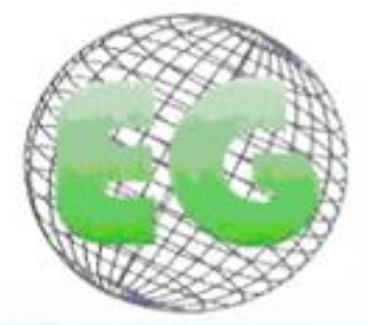

www.um.es/egloball

\title{
CLÍNICA
}

\section{Consumo de drogas psicotrópicas y capacidades de autocuidado en mujeres de Tepic, Nayarit}

Psychotropic drug consumption and self-caring capabilities of women in Tepic, Nayarit

*Valdivia Pérez, Luis Gerardo **Casique Casique, Leticia ***Muñoz Torres,

\section{Teresita de Jesús}

\begin{abstract}
*Maestro en Ciencias de Enfermería, docente de la Universidad Autónoma de Nayarit. **Doctora en Ciencias de Enfermería, docente e Investigador de la Facultad de Enfermería y Obstetricia de Celaya, Universidad de Guanajuato. E-mail: leticiacc_2004@yahoo.com.mx ***Licenciada en Enfermería, docente de la Unidad Académica Multidisciplinaria Zona Media de la UASLP. México.
\end{abstract}

Palabras Clave: Drogas Psicotrópicas; Capacidad de autocuidado; Mujeres.

Keywords: Psychotropic drugs; ability for self-care; women.

\section{RESUMEN}

Objetivo: Determinar la asociación entre el consumo de drogas psicotrópicas y la capacidad de autocuidado en las mujeres.

Material y métodos: Estudio no experimental, cuantitativo, aplicado, prolectivo y transversal. La muestra estuvo conformada por 33 mujeres que consumen drogas psicotrópicas de la colonia Tierra y Libertad de Tepic, Nayarit.

Resultados: El $73 \%$ son solteras, $51.5 \%$ tiene depresión, $21.2 \%$ insomnio y $15.2 \%$ ansiedad. El $69.7 \%$ reportó un tiempo de consumo de drogas psicotrópicas de 1 a 5 años. El $75.8 \%$ no realiza técnicas para controlar la depresión, estrés o ansiedad; $78.8 \%$ no realiza algún ejercicio, $54.5 \%$ no saben las consecuencias de las drogas psicotrópicas, $48.5 \%$ no resuelve los problemas que se le presentan cotidianamente en la vida y el $36.4 \%$ no le agrada convivir con su familia y amigos. Se encontró un alto nivel de conocimientos $64 \%$ y un nivel bajo de habilidades y actitudes en el $82 \%$ y $53 \%$ respectivamente. Al aplicar índice eta $(\eta)$ con una $p<0.05$ entre las capacidades de autocuidado y el consumo de drogas psicotrópicas, se encontró una asociación y diferencia entre estas dos variables, aceptándose la hipótesis de investigación e hipótesis alterna.

Conclusiones: Existe una asociación y diferencias significativas entre las variables capacidades de autocuidado y el consumo de drogas psicotrópicas. 


\section{ABSTRACT}

Objective: To determine the association between the consumption of psychotropic drugs and the capabilities of self-care in women.

Materials and Methods: a non-experimental, quantitative, applied, simultaneous, and cross-sectional study was carried out. The sample was made up of 33 women that consume psychotropic drugs in the Tierra and Libertad neighborhoods in Tepic, Nayarit.

Results: Of those studied, $73 \%$ were single, $51.5 \%$ suffer with depression, $21.2 \%$ suffer with insomnia and $15.2 \%$ with anxiety. More than half $(69.7 \%)$ reported a duration of psychotropic drug consumption of between 1 and 5 years. The majority $(75.8 \%)$ did not use any techniques to control depression, stress, nor anxiety, and $78.8 \%$ did not do any exercise. Just over half $(54.5 \%)$ were not aware of the consequences of psychotropic drug use, $48.5 \%$ do not resolve daily life problems, and $36.4 \%$ do not like living with friends and family. We found a high level of knowledge $(64 \%)$ and a low level of skills and attitudes (82\% and 53\% respectively). By applying the eta $(n)$ index with $p<0.05$ between the self-care skills and the consumption of psychotropic drugs, an association and difference between these variables was find, leading to the acceptance of the hypothesis and the alternative hypothesis.

Conclusions: There are significant associations and differences between the variables in the self-care ability and the consumption of psychotropic drugs.

\section{INTRODUCCIÓN}

El aumento del consumo de drogas psicotrópicas en mujeres de Latinoamérica se atribuye a factores tales como la mayor exposición y acceso a las drogas, falta de información o una información equivocada sobre el consumo de las mismas, presiones de parejas, necesidad de escapar o enfrentar la realidad de la pobreza, opresión, violencia doméstica y abuso sexual en la niñez. Todos estos aspectos se encuentran de alguna manera relacionados con el cambio de roles y aumento de responsabilidades en la mujer ${ }^{(1)}$.

Hasta la fecha se ha prestado poca atención a los temas relacionados con las mujeres en las capacidades de autocuidado y el uso y abuso de drogas psicotrópicas. La mayor parte de los esfuerzos de prevención y rehabilitación en salud están dirigidos a la población joven, especialmente a varones; mientras que las iniciativas para las mujeres se centran tanto a nivel nacional como regional en la salud reproductiva, materno-infantil y los problemas relacionados con la violencia contra la mujer.

La población de mujeres entre 20 y 40 años culturalmente es un grupo vulnerable por las características propias de género, pues estas suelen estar ansiosas o deprimidas por los múltiples roles que desempeñan en el transcurso de la vida; esta situación propicia que se presenten problemas de salud físicos y emocionales, aumentando los índices de atención y el riesgo de automedicación ${ }^{(2)}$.

En el contexto internacional las cifras estadísticas sobre el consumo de drogas psicotrópicas refieren un índice de seis veces superiores a Estados Unidos de América, en Argentina alcanza 2.3\%, en Chile 2.1\%, en Colombia 1.2\%, en Perú 1\% y en Brasil $0.8 \%$. En países europeos como Alemania (2.1\%) y Holanda (1.8\%). En México se ubica entre aquellos países que tienen bajas tasas de consumo de drogas psicotrópicas, pero que a su vez, reportan incremento del problema. El índice de dependencia se encuentra $(0.7 \%)$, por su parte, la Organización de las Naciones Unidas (ONU) estima una prevalencia mundial de $4.2 \%$ para cualquier droga médica 
en la población de 15 años en adelante, en tanto en México el índice para la población de 12 a 65 años de edad ha aumentado de un $5 \%$ en el 2002 a un $6 \%$ del $2008{ }^{(3)}$.

Por lo anterior el consumo de drogas psicotrópicas en el país constituye uno de los principales problemas de salud pública por el abuso y dependencia de sustancias médicas. Este fenómeno a nivel mundial, incluyendo México, va en aumento, debido a que tanto los países productores de drogas como los de tránsito se convierten en consumidores, lo que genera mayores problemas de salud, de gobernabilidad, de cumplimiento de las leyes, de convivencia y de paz social. Por tal motivo, se puede afirmar que el consumo de drogas psicotrópicas en las mujeres se ha agravado en los últimos años, debido al proceso de globalización, factores políticos, económicos, socioculturales, esto aunado a las responsabilidades y a los problemas familiares.

La Encuesta Nacional de Adicciones (ENA) realizada en México en 2008, ha permitido identificar el porcentaje en el consumo de cualquier droga médica, reportando un $6.0 \%$. En su mayoría, las drogas empleadas son fármacos adquiridos con receta médica, por ello los fármacos más utilizados por las mujeres son las drogas psicotrópicas (diazepam y flunitrazepam). También se ha empezado a detectar el uso de la Burundanga, una hierba con efecto tranquilizante.

Respecto al género, en algunos estudios recientes se ha observado diferencias en el consumo de drogas. Por ejemplo, en poblaciones de adultos mayores se reporta que los hombres tienden a consumir en mayor cantidad y frecuencia el alcohol que las mujeres, y en relación con el consumo de drogas psicotrópicas las mujeres son las que consumen en mayor cantidad y con mayor frecuencia que los hombres ${ }^{(4)}$.

Sabiendo de la problemática que está presentando la mujer sobre el consumo de drogas psicotrópicas, se realizó este estudio para conocer la asociación entre el consumo de drogas psicotrópicas y la capacidad de autocuidado en las mujeres de la colonia Tierra y Libertad de la ciudad de Tepic, Nayarit. Por lo antes descrito es que este articulo retoma las aportaciones teóricas de Dorothea Orem plasmadas en la Teoría del Déficit de Autocuidado, en donde describe que la persona tiene una capacidad de autocuidado si las habilidades desarrolladas permiten a la persona realizar de manera efectiva su cuidado, dentro de un marco temporal adecuado para mantener el funcionamiento y desarrollo dentro de las normas compatibles con la vida, la salud y el bienestar ${ }^{(5)}$.

\section{MATERIAL Y MÉTODOS}

\section{Tipo de estudio}

Estudio no experimental, cuantitativo, correlacional, aplicativo y de corte transversal. Realizado de Enero del 2009 a Julio 2010 en la colonia Tierra y Libertad de Tepic Nayarit, México; en mujeres entre 25 y 45 años consumidoras de drogas psicotrópicas.

\section{Muestra}

Se tomó una muestra total de 33 mujeres entre 25 y 45 años que consumen Drogas Psicotrópicas de la colonia Tierra y Libertad de Tepic Nayarit, misma que representó el $100 \%$ del universo de estudio. La muestra fue seleccionada por disponibilidad. 


\section{Instrumentos}

Para la obtención de los datos se utilizó un instrumento exprofeso denominado Cuestionario sobre las Capacidades en las Mujeres que Consumen Drogas Psicotrópicas; el instrumento está compuesto de 4 apartados: Datos de Identificación, Drogas Psicotrópicas, Consumo de drogas psicotrópicas y Capacidades de Autocuidado; el cuestionario obtuvo un valor de alfa de cronbach de 0.80 .

En el apartado de datos de identificación se encuentran 5 variables sociodemográficas (edad, escolaridad, ocupación, estado civil, religión); el segundo apartado, drogas psicotrópicas, se divide en dos secciones, la primera sobre datos básicos descriptivos relacionados con el consumo de drogas psicotrópicas y la segunda sobre el consumo de drogas psicotrópicas. La tercera sección consta de 4 categorías, cada una de 3 preguntas para medir la dosis de consumo diario, semanal y mensual con un rango de valores del 1 al 4 , si la respuesta es 1 o 2 se califica como dosis baja y si es de 3 o 4 como dosis alta. Por último, el apartado de capacidades de autocuidado incluye 17 preguntas divididas en conocimiento, habilidad y actitud medidas con una escala dicotómica $(1=\mathrm{No}, 2=\mathrm{Si})$; la suma de las puntuaciones de los tres apartados nos da la medición total de la capacidad de autocuidado, para ello, se establece bajo conocimiento y actitudes si la puntuación total es de 6 a 9 y alto conocimiento y actitudes con una puntuación de 10 a 12, mientras que la habilidad se determina como baja si la puntación es de 5 a 7 y alta si la suma total es 8 a 10 .

\section{Análisis de datos}

Se utilizó el Paquete Estadístico para las Ciencias Sociales SPSS versión 17, el análisis contemplo el uso de tablas de frecuencia y se efectúo un cruce de variables por medio de tablas de contingencia para verificar la dependencia entre ellas, para comprobar la hipótesis y la asociación entre el consumo de drogas psicotrópicas y las capacidades de autocuidado; por último, se realizó un cruce de cada una de las drogas consumidas con el nivel de las capacidades de autocuidado, para obtener el coeficiente eta $(\eta)$, donde se estableció como valores de .0 a .30 para una baja asociación, de .31 a .50 media asociación y de .51 a 1 alta asociación; la prueba eta (n) se utiliza para analizar una variable nominal y una variable de escala.

\section{RESULTADOS}

La tabla 1 muestra los resultados obtenidos de la muestra objeto de estudio en relación con los aspectos sociodemográficos, en donde se identifica como dato relevante que el $73 \%$ de las mujeres encuestadas son solteras, dato que coincide con la ocupación que desempeñan (empleadas), es decir, que ejercen el rol de proveedor para el sustento familiar, lo cual puede generar una presión familiar y una alteración emocional lo que repercute en el inicio del consumo de drogas psicotrópicas.

Tabla 1. Perfil de las Mujeres que consumen drogas psicotrópicas de Tepic, Nayarit 2010

\begin{tabular}{||l||l||c||c||}
\hline VARIABLE & \multicolumn{1}{|c|}{ CATEGORIAS } & F & $\%$ \\
\hline \hline \multirow{5}{*}{ Edad } & 25 a 30 años & 6 & $18.2 \%$ \\
\cline { 2 - 4 } & 31 a 35 años & 4 & $12.1 \%$ \\
\cline { 2 - 4 } & 36 a 40 años & $\mathbf{1 3}$ & $39.4 \%$ \\
\cline { 2 - 4 } & 41 a 45 años & $\mathbf{1 0}$ & $\mathbf{3 0 . 3} \%$ \\
\hline
\end{tabular}




\begin{tabular}{|c|c|c|c|}
\hline \multirow{7}{*}{ Escolaridad } & Analfabeta & 1 & $3.0 \%$ \\
\hline & Primaria incompleta & 3 & $9.1 \%$ \\
\hline & Primaria Completa & 10 & $30.3 \%$ \\
\hline & Secundaria Incompleta & 3 & $9.1 \%$ \\
\hline & Secundaria Completa & 8 & $24.2 \%$ \\
\hline & Preparatoria & 7 & $21.2 \%$ \\
\hline & Profesional & 1 & $3.0 \%$ \\
\hline \multirow{7}{*}{ Ocupación } & Estudiante & 2 & 6.1 \\
\hline & Ama de Casa & 6 & 18.2 \\
\hline & Obrera & 0 & $0 \%$ \\
\hline & Comerciante & 1 & 3.0 \\
\hline & Empleada & 24 & 72.7 \\
\hline & Desempleada & 0 & $0 \%$ \\
\hline & Otros Especifique & 0 & $0 \%$ \\
\hline \multirow{5}{*}{ Edo. Civil } & Soltera & 24 & $73 \%$ \\
\hline & Casada & 7 & $21 \%$ \\
\hline & Unión Libre & 0 & $0 \%$ \\
\hline & Divorciada & 1 & $3 \%$ \\
\hline & Viuda & 1 & $3 \%$ \\
\hline \multirow{5}{*}{ Religión } & Católica & 33 & $100 \%$ \\
\hline & Cristiana & 0 & $0 \%$ \\
\hline & Testigo de Jehová & 0 & $0 \%$ \\
\hline & Atea & 0 & $0 \%$ \\
\hline & Otros Especifique & 0 & $0 \%$ \\
\hline \multirow{5}{*}{ Institución } & Salubridad & 9 & $27.3 \%$ \\
\hline & Seguro Popular & 8 & $24.2 \%$ \\
\hline & ISSSTE & 1 & $3.0 \%$ \\
\hline & IMSS & 15 & $45.5 \%$ \\
\hline & Particular & 0 & $0 \%$ \\
\hline \multirow{5}{*}{ Diagnóstico } & Ansiedad & 5 & $15.2 \%$ \\
\hline & Pánico & 0 & $0 \%$ \\
\hline & Depresión & 17 & $51.5 \%$ \\
\hline & Problemas de Sueño & 7 & $21.2 \%$ \\
\hline & Estrés & 4 & $12.1 \%$ \\
\hline
\end{tabular}

Fuentes: Cuestionario Consumo de Drogas Psicotrópica

Otro dato sobresaliente según el diagnóstico médico es que el $51.5 \%$ presenta problemas de depresión; $15.2 \%$ ansiedad, $12.1 \%$ estrés y el $21.2 \%$ problemas de sueño, lo que refiere que la mujer presenta una alteración en la estabilidad emocional y que no tiene mecanismos para enfrentar su problemática emocional, por lo que se apoyan en el consumo de drogas psicotrópicas, esto sin duda es un dato que debe de impactar en el cuidado de enfermería.

En relación al consumo de drogas psicotrópicas, la tabla 2 demuestra un consumo preferido por el diazepam, con un tiempo de consumo menor de un año y mayor de 5 años. Las drogas que se registran en este estudio pertenecen a la clase II de las drogas psicotrópicas donde se ubican los ansiolíticos y tranquilizantes, lo cual refleja que las mujeres padecen de ansiedad, intranquilidad y depresión. 
Tabla 2. Drogas psicotrópicas que consumen las mujeres

Tepic, Nayarit 2010

\begin{tabular}{|c|c|c|}
\hline VARIABLES & $\bar{f}$ & $\%$ \\
\hline \multicolumn{3}{|l|}{ Drogas Psicotrópicas } \\
\hline Diazepam & 12 & $36.4 \%$ \\
\hline Clonazepam & 8 & $24.2 \%$ \\
\hline Lorazepam & 8 & $24.2 \%$ \\
\hline Flunitrazepam & 5 & $15.2 \%$ \\
\hline \multicolumn{3}{|l|}{ Tiempo de Consumo } \\
\hline$<$ de 1 año & 9 & $27.3 \%$ \\
\hline 1 a 3 años & 8 & $24.2 \%$ \\
\hline 4 a 5 años & 6 & $18.2 \%$ \\
\hline$>$ de 5 años & 10 & $30.3 \%$ \\
\hline \multicolumn{3}{|c|}{ Persona que le Índico el Medicamento } \\
\hline Médico & 27 & $81.8 \%$ \\
\hline Familiar & 1 & $3.0 \%$ \\
\hline Farmacia & 4 & $12.1 \%$ \\
\hline Trabajo & 1 & $3.0 \%$ \\
\hline \multicolumn{3}{|c|}{ Solicitud de Receta Médica } \\
\hline Si & 24 & $72.7 \%$ \\
\hline No & 9 & $27.3 \%$ \\
\hline
\end{tabular}

Fuentes: Cuestionario Consumo de Drogas Psicotrópica

El estudio establece un consumo alto de drogas psicotrópicas, ya que las mujeres que consumen diazepam registraron un consumo por día, semana y mes de $54 \%$ y $100 \%$, respectivamente; mientras que el clonazepam registró que el 100\% de las mujeres lo consumen en estas dimensiones días, semana y mes; las mujeres que consumieron lorazepam señalaron un consumo por día y semana bajo $(80 \%)$ pero un consumo mensual alto en un $100 \%$, por su parte, el flunitrazepam es consumido al día, semana y mes con un valor de $86 \%$ y un $100 \%$ lo que indica un consumo alto; esto permite establecer que las 33 mujeres entrevistadas tienen altas probabilidades de desarrollar síndrome de tolerancia fisiológica y psicológica.

Al aplicar la escala de capacidad de autocuidado se encontró que más del $50 \%$ posee conocimientos sobre el consumo de drogas psicotrópicas, pues el (63.6\%) conoce el medicamento que está tomando y los efectos de este medicamento, el $75.8 \%$ conoce los síntomas que le indican que su cuerpo está en buen estado de salud, el $69.7 \%$ conoce los cambios que produce cuando no se toma el medicamento, el $51.5 \%$ conoce la dosis correcta y el $60.6 \%$ conoce los beneficios del ejercicio. Esto indica que las 33 mujeres poseen alto conocimiento respecto a la causa, dosis, síntomas y efectos que tienen las drogas psicotrópicas que consumen, lo que indica que han recibido una orientación de la farmacología del medicamento; lo cual coincide con Orem ya que las mujeres deben de poseer un repertorio de conocimiento para poder llevar su autocuidado. 
Sin embargo al ser cuestionadas sobre las habilidades que ejecutan para su cuidado, resalta un porcentaje alto de mujeres que no realizan prácticas y/o técnicas cuando se sienten ansiosas o deprimidas (75.8\%), no suelen realizar deportes $(78.8 \%)$, no realizan ejercicio para disminuir la ansiedad (84.8\%) y un $90.9 \%$ de las mujeres registraron no realizar ejercicios entre 20 a 30 minutos tres veces por semana como mínimo. Estos porcentajes reflejaron efectivamente que las mujeres conocen su diagnóstico, la farmacología de las drogas psicotrópicas que consumen, pero no realizan ningún deporte o actividad física para controlar la ansiedad y la depresión; esta situación se puede interpretar por las condiciones socioeconómicas que tienen las mujeres, es decir, son solteras, empleadas y tienen de dos a tres hijos.

En relación a los resultados obtenidos sobre las actitudes que poseen las mujeres que consumen drogas psicotrópicas son favorables, pues se comunican fácilmente con sus seres queridos y resuelven problemas que se les presentan en un $51.5 \%$, les agrada convivir con sus familiares y amigos (63.6\%), y respetan sus valores de vida (63.6\%). Sin embargo al $69.7 \%$ no le gusta participar con el apoyo de un instructor en actividades físicas ni tener apoyo psicológico.

Al determinar el nivel total de capacidad de autocuidado en las mujeres que consumen drogas psicotrópicas; se registró un nivel alto de conocimiento con un porcentaje de $64 \%$, relacionado con las habilidades se encontró un nivel bajo (82\%) y bajo nivel de actitud con un $52 \%$ (tabla 3 ).

Tabla 3. Nivel de capacidades de autocuidado Tepic Nayarit 2010

\begin{tabular}{|l|l|c|c|}
\hline \multicolumn{2}{|c|}{$\begin{array}{c}\text { CAPACIDAD DE } \\
\text { AUTOCUIDADO }\end{array}$} & F & $\%$ \\
\hline \hline \multirow{3}{*}{$\begin{array}{l}\text { NIVEL DE } \\
\text { CONOCIMIENTO }\end{array}$} & Bajo & 12 & $36 \%$ \\
\cline { 2 - 4 } & Alto & $\mathbf{2 1}$ & $\mathbf{6 4 \%}$ \\
\cline { 2 - 4 } & Total & 33 & $100 \%$ \\
\hline \hline \multirow{3}{*}{ NIVEL DE HABILIDAD } & Bajo & $\mathbf{2 7}$ & $\mathbf{8 2} \%$ \\
\cline { 2 - 4 } & Alto & 6 & $18 \%$ \\
\cline { 2 - 4 } & Total & 33 & $100 \%$ \\
\hline \hline \multirow{3}{*}{ NIVEL DE ACTITUD } & Bajo & $\mathbf{1 7}$ & $\mathbf{5 2 \%}$ \\
\cline { 2 - 4 } & Alto & 16 & $48 \%$ \\
\cline { 2 - 4 } & Total & 33 & $100 \%$ \\
\hline
\end{tabular}

Fuentes: Cuestionario de Capacidad de Autocuidado

Para comprobar la hipótesis se utilizó el índice eta (n) para valorar la asociación entre capacidades de autocuidado con el consumo de drogas psicotrópicas aun nivel de significancia de <.05. La tabla 4 demuestra los resultados que se encontraron entre el consumo de drogas psicotrópicas (diazepam, clonazepam, lorazepam y flunitrazepam) con las capacidades de autocuidado (nivel de conocimiento, nivel de habilidades y nivel de actitud y/o motivación), y en donde se identifica que existe una asociación entre las variables de estudio. Sin embargo, cabe aclarar que existió una 
mayor asociación entre el diazepam y lorazepam que el clonazepam y el flunitrazepam con las capacidades de autocuidado; esto se explica porque el mayor consumo de drogas psicotrópicas fue el diazepam (36.4\%) y lorazepam (24.2\%).

Tabla 4. Asociación entre capacidades de autocuidado y consumo de drogas psicotrópicas

Tepic, Nayarit 2010

\begin{tabular}{||c|c|c|c|c||c|c||}
\hline \multicolumn{7}{|c|}{ TABLA RESUMEN DE ETA ( $)$} \\
\hline $\begin{array}{c}\text { CAPACIDAD DE } \\
\text { AUTOCUIDADO }\end{array}$ & \multicolumn{2}{|c|}{ CONOCIMIENTO } & \multicolumn{2}{c||}{ HABILIDAD } & \multicolumn{2}{c|}{ ACTITUD } \\
\hline \hline MEDICAMENTO & $\mathrm{H}$ & $\mathrm{p}$ & $\eta$ & $\mathrm{P}$ & $\eta$ & $\mathrm{p}$ \\
\hline \hline DIAZEPAM & .33 & $<.05$ & .41 & $<.05$ & .14 & $<.05$ \\
\hline \hline CLONAZEPAM & .29 & $<.05$ & .14 & $<.05$ & .13 & $<.05$ \\
\hline \hline LORAZEPAM & .31 & $<.05$ & .31 & $<.05$ & .16 & $<.05$ \\
\hline \hline FLUNITRAZEPAM & .14 & $<.05$ & .24 & $<.05$ & .20 & $<.05$ \\
\hline
\end{tabular}

Fuentes: Asociación entre Capacidad de Autocuidado y Consumo de Drogas Psicotrópicas

\section{DISCUSIÓN}

Según los resultados, las mujeres tienen educación básica $(72.7 \%)$, son empleadas $(72.7 \%)$, solteras $(73 \%)$, presentan depresión $(51.5 \%)$, problemas del sueño $(21.2 \%)$, ansiedad $(15.2 \%)$ y estrés $(12.1 \%)$; estos resultados coinciden con los obtenidos en otras investigaciones, en donde el inicio de consumo de drogas psicotrópicas fue como recurso para hacerle frente a los factores estresores de la vida cotidiana, como ansiedad en un $30 \%$ e inductores de sueño en el $46 \%{ }^{(6)}$.

Los resultados sobre el consumo de drogas psicotrópicas según día y semana reportan un consumo alto excepto el lorazepam que registró solamente un consumo alto durante el mes; esta situación aunada al tiempo de consumo de más de 5 años $(72.7 \%)$ confirman que las mujeres han desarrollado el síndrome de tolerancia. Estos resultados concuerdan con los descritos por Secades en donde el $80 \%$ de la población estudiada consume ansiolíticos, de los cuales el 30\% consume lorazepam. Del $80 \%$ mencionado anteriormente se presenta dependencia en el $37.9 \%$ relacionada con el tiempo de consumo entre 1 y 5 años, los autores consideran que la diferencia entre los consumidores de más de un año y los de 5 años no es significativa con respecto a la dependencia ${ }^{(7)}$.

Por su parte, en un estudio realizado en Monterrey Nuevo León en el año 2008 reporta que el consumo de drogas médicas por género fue en adultos mayores, en donde el $20 \%$ de las mujeres las consume mientras que solo un $4.4 \%$ de los hombres reportó estar consumiéndolos, estas proporciones mostraron ser significativamente diferentes $\left(X^{2}=5.95, p=.015\right)$. Respecto al tipo de droga médica reportado, se destacó el tranquilizante (ansiolítico) con $83.5 \%$ y antidepresivos $16.5 \%$. Otro dato relevante 
es que más del $30 \%$ de las veces se consiguió esta clase de medicamentos sin receta médica ${ }^{(8)}$.

Sin embargo en el presente estudio el $12.1 \%$ señaló que la persona que le indicó la droga psicotrópica fue en la farmacia, sin especificar si fue un médico o un empleado. Además se encontró que en el $18.2 \%$ de las mujeres la indicación de las drogas psicotrópicas fue por un familiar, en la farmacia y/o en el trabajo; señalando además que al comprar la droga psicotrópica en la farmacia no le solicitaban receta médica (27.3\%). Esta situación va en contra de lo establecido por la Ley General de Salud en el capítulo IV, artículo 226 que establece que "las drogas según los efectos que produce en el SNC es considerado un medicamento controlado, sustancia que según su potencial de abuso y adicción debe respetar condiciones para su venta" (9). Estos resultados coinciden con los datos establecidos en una publicación del 2007 en donde refieren que en el $12.1 \%$ de las personas que consumen drogas psicotrópicas, no existe un control en su consumo y venta ${ }^{(10)}$.

Al aplicar el índice eta (n) entre las variables de estudio, se encontró que existe mayor asociación entre el diazepam y el lorazepam con el nivel de conocimiento, ya que se encontró que un $36.4 \%$ consume diazepam y lorazepam en un $24.2 \%$, estos resultados presentan una concordancia con un estudio realizado en el 2006 en donde se establece que en la automedicación psiquiátrica es más común el uso de sedantes e hipnóticos, en donde del $100 \%$, un $48.6 \%$ consume diazepam y lorazepam con un $10.8 \%$. Lo que indica que estas drogas psicotrópicas son las más conocidas por la población, como recurso frente a la depresión, insomnio, tristeza y ansiedad ${ }^{(11)}$.

Con respeto a las capacidades se encontró un nivel alto de conocimientos $64 \%$ y un nivel bajo de habilidades y actitudes de $82 \%$ y $52 \%$ respectivamente, el $75.8 \%$ no realiza ninguna práctica o técnica para controlar su depresión, estrés o ansiedad, en relación de que si saben las consecuencias de las drogas psicotrópicas respondió que no un $54.5 \%$, estos resultados coinciden con los reportados en una investigación realizada en el 2010, en donde evidencian que la variable de Conocimiento obtuvo la mayor proporción de los elementos muéstrales y ubicaron sus respuesta en un nivel intermedio para el manejo de sustancias psicotrópicas lo que correspondió a un $(63.3 \%)$ de la muestra. Con relación a la variable Actitud en la administración de sustancias psicotrópicas las enfermeras demostraron una actitud con un nivel alto de un $(96.7 \%)$ de la muestra estudiada con una $p<0.05$, no existiendo correlación entre la variable actitud y conocimiento de estas profesionales al igual que en el presente estudio ${ }^{(12)}$.

\section{CONCLUSIONES}

Al aplicar el índice eta $(\eta)$ con una $p<0.05$ entre las capacidades de autocuidado con el consumo de drogas psicotrópicas; se encontró una asociación entre estas dos variables aceptando la hipótesis de investigación. Sin embargo, se encontró diferencias en el consumo de drogas psicotrópicas entre las mujeres con las capacidades de autocuidado, aceptándose a su vez la hipótesis alterna.

Los datos descriptivos reflejan que efectivamente las mujeres están teniendo un consumo alto de estas drogas psicotrópicas que puede provocar una adicción dependiente y el desarrollar síndrome de tolerancia al igual que disminuyen sus capacidades de autocuidado lo cual afecta la salud de la mujer y la salud familiar debido a que actualmente la mujer cumple el rol de proveedor (empleada y soltera). 
De ahí la importancia de realizar seguimiento de los casos en mujeres que consumen drogas psicotrópicas por grupo de edad.

Por tal motivo es importante dar a conocer estos resultados a los programas correspondientes de la Secretaria de Salud para dar seguimiento y que el profesional de enfermería intervenga de manera oportuna para detectar el consumo de dichas sustancias y solicitar el apoyo multidisciplinario para fortalecer las capacidades de autocuidado evitando la adicción a estas drogas psicotrópicas y a su vez favorecer el bienestar físico, psicológico y social.

\section{REFERENCIAS}

1. Sonita Marin A. El impacto del Abuso de Drogas en las Mujeres y sus Familias. Uruguay: Addiction Alert Organization Montevideo; 1966. Disponible en línea http://www.cicad.oas.org/Reduccion_Demanda/esp/Mujer/jamaica.pdf> (Recuperado 15 de Agosto de 2013).

2. Romero M. Discapacidad en Mujeres Adictas Encarceladas, Inequidad Social o Problema Personal. México: Instituto Nacional de Psiquiatría Ramón de la Fuente; 2002. Disponible en línea http://www.buap.mx/vision/discap/p_251.doc (Recuperado 15 de Agosto de 2013).

3. Medina M; et al. Consumo de Drogas entre Adolescentes: Resultados de la Encuesta Nacional de Adicciones. Salud Pública de México. 2003; 45 (Sup 1):16-25.

4. Medina Mora ME; Rojas Guiot E. Mujer, Pobreza y Adicciones. Perinatol reprod Hum. 2003; 17: 230-244.

5. Marriner A, Raile M. Modelos y teorías en enfermería. 7aㅡ ed. España: Elsevier Mosby; 2007.

6. Ávila K. Consumo de sustancias psicoactivas capaces de producir dependencia. Costa Rica: Centro de desarrollo estratégico Universidad de Costa Rica; 2003. Disponible en línea www.cendeisss.sa.cr/modulos/AISmodulo3.pdf (Recuperado 15 de Agosto de 2013).

7. Secades Villa R; Rodríguez García E; Valderrey Barbero J; Fernández Hermida JR; Vallejo Seco G; Jiménez García JM. El consumo de psicofármacos en pacientes que acuden a Atención Primaria en el principiado de Asturias. Psicothema 2003; 15 (4): 650-655.

8. Alonso Castillo BA; Palucci Marziale MH; Alonso Castillo MM; Guzmán Facundo FR; Gómez Meza MV. Situaciones de la vida estresante, uso y abuso de alcohol y drogas en adultos mayores de Monterrey, México. Revista Latino-Americana de Enfermagem, 2008; 16.

9. Secretaria de Salud. Norma Oficial Mexicana NOM-028-SSA2-2009, Para la prevención, tratamiento y control de las adicciones. Disponible en línea http://www.conadic.salud.gob.mx/pdfs/publicaciones/nom028.pdf (Recuperado 15 de Agosto de 2013).

10. Hernández Espallardo M; Delgado Ballester E; Munuera Alemán JL. Elección del Lugar de Compra de Medicamentos y parafarmacia. España; 2007. Disponible en línea www.dialnet.unirioja.es/servlet/articulo?codigo=20785 (Recuperado 15 de Agosto de 2013).

11. Palacios F. Automedicación Psiquiátrica [Tesis Maestría].Hospital Docente de Atención Psicosocial. Universidad Nacional Autónoma de Nicaragua; 2006.

12. Mark E. Conocimientos y actitudes que tiene el personal de enfermería en el manejo de sustancias psicotrópicas la Unidad de Cuidados Intensivos, Facultad 
de Ciencias de la Salud, Escuela de Enfermería. Venezuela; 2010. Disponible en línea http://www.portalesmedicos.com/publicaciones/articles/2184/1/Conocimiento-yactitud-que-tiene-el-personal-de-Enfermeria-en-el-manejo-de-sustanciaspsicotropicas-en-la-Unidad-de-Cuidados-Intensivos.html> (Recuperado 15 de Agosto de 2013).

Recibido: 17 de agosto de 2013; Aceptado: 23 de octubre de 2013 